\title{
Knowledge, attitude and practice regarding emergency contraceptive pills among female health workers of a tertiary care hospital in Karachi
}

\author{
Mubashir Ahmed ${ }^{1}$, Farah $\mathrm{Naz}^{2}$, Khalid Iqbal $^{3}$, Ashraf Memon ${ }^{4}$
}

\author{
${ }^{1}$ Department of Research, Kharadar General Hospital, Aga Khan Road, Kharadar, Karachi, Pakistan \\ ${ }^{2}$ Department of Obstetrics and Gynecology, Kharadar General Hospital, Aga Khan Road, Kharadar, Karachi, Pakistan \\ ${ }^{3}$ Chief Executive Officer, Kharadar General Hospital, Karachi, Sindh, Pakistan \\ ${ }^{4}$ Department of Pathologist, Kharadar General Hospital, Karachi, Sindh, Pakistan
}

Received: 27 July 2016

Accepted: 28 August 2016

\section{*Correspondence:}

Dr. Mubashir Ahmed,

E-mail: Mubashir.ahmed71@gmail.com

Copyright: (C) the author(s), publisher and licensee Medip Academy. This is an open-access article distributed under the terms of the Creative Commons Attribution Non-Commercial License, which permits unrestricted non-commercial use, distribution, and reproduction in any medium, provided the original work is properly cited.

\section{ABSTRACT}

Background: Unsafe abortion is a major public health problem in low-and-middle income countries. Young and unmarried women constitute a high risk group for unsafe abortions. Use of emergency contraceptive pills (ECPs) may significantly reduce the abortion-related morbidity and mortality. The purpose of this study was to evaluate the knowledge, attitudes and practice (KAP) about ECPs among female health workers of a tertiary care hospital in Karachi.

Methods: In this hospital based cross-sectional study, 187 female health workers (nurses and midwives) were participated. A predesigned, 26-item questionnaire was used to collect participants' responses regarding knowledge, attitude and practice of ECP. Descriptive analysis of data was done by using SPSS-version 16.

Results: Total 187 female health workers (95 nurses and 92 midwives) were participated in the study. Mean age of study participants was $23.5 \pm 7.31$. Mean knowledge score of the study participants was $3.7 \pm 2.18$ (Out of score: $1-10$ ). Majority participants $151(80.7 \%)$ had inadequate knowledge (score $\leq 50 \%$ ) about ECP. The mean attitude score of the study participants was $20.9 \pm 2.68$ (Out of score: $8-32$ ). Significant number $84(45 \%)$ of participants had negative (score $\leq 50 \%$ ) attitudes towards ECPs.

Conclusions: There was gap in knowledge and negative attitude towards ECP were identified among female health workers. There was a need to bridge the gap of knowledge on ECP and remove the misconception about the use and safety of ECP among female health workers.

Keywords: Attitude, Emergency contraceptive pills, Female health workers, Knowledge, Practice

\section{INTRODUCTION}

Unwanted pregnancy and unsafe abortion is a major public health problem in developing countries. It has been estimated that use of emergency contraception (EC) may significantly reduce the number of abortion-related morbidity and mortality. ${ }^{1}$ The World Health Organization (WHO) estimates that 84 million unwanted pregnancies occur annually worldwide. ${ }^{2}$ On average, 46 million abortions take place every year, out of which 20 million were performed under unsafe conditions., ${ }^{2,3}$ As a consequence of unsafe abortion nearly 70,000 women die and 5 million suffer permanent or temporary disability every year. $^{2-4}$ Globally $13 \%$ of pregnancy-related maternal mortality occurs due to unsafe abortions and majority of these deaths occur in low-and-middle income countries. ${ }^{5,6}$ In Pakistan unwanted pregnancy rate is 37 percent of all pregnancies or 29 per 1,000 women of reproductive aged 15-49 years. $^{7}$ Estimated 890,000 induced abortions occurs every year and abortion account for termination of one in seven pregnancies. ${ }^{7}$ Attention 
has been focused on the potential of ECPs to reduce the burden of unwanted pregnancies and abortion. ${ }^{8}$

Emergency contraceptive pill (ECP) is used to reduce unwanted pregnancy while used within 72-120 hours of unprotected sexual intercourse. Conditions during which women may use ECP includes; when no contraceptive method is used, failure of natural withdrawal or modern contraceptive methods like slippage or breakage of condom, missing of three or more consecutive oral contraceptive pills (OCPs), delay of more than two weeks in injectable methods, expulsion of intra uterine contraceptive device (IUCD) and in case of sexual assault. ${ }^{9}$ Mechanism of action of ECP was to inhibit or delay ovulation. ${ }^{10-12}$ Additional possible mechanisms of action include interference with corpus luteum function; thickening of the cervical mucus resulting in trapping of sperm; alterations in the tubal transport of sperm, egg or embryo and direct inhibition of fertilization. ${ }^{13-14}$

There are two common types of hormonal ECPs: First, combined ECPs containing both estrogen and progestin (Yuzpe regimen) and second, progestin-only ECP. ${ }^{15}$ Yuzpe regimen administered as two doses of combined oral contraceptive pills (each dose containing $100 \mu \mathrm{g}$ of ethinyl estradiol and $1 \mathrm{mg}$ of norgestrel) taken 12 hours apart within 72-120 hours of the unprotected sex. ${ }^{16}$ Whereas Progestin-only ECPs administered as two doses of progestin levonorgestrel (each dose of $0.75 \mathrm{mg}$ ) 12 hours apart or taken as a single dose $(1.5 \mathrm{mg})$ after unprotected sex. ${ }^{17}$ However, studies have shown that a single dose of $1.5 \mathrm{mg}$ levonorgestrel is as effective as two $0.75 \mathrm{mg}$ doses taken 12 hours apart. Study also reported that progestin only pills would reduce the chance of pregnancy by $85 \%$, and combined hormone EC pills by $75 \%$ when taken within 72 hours of unprotected sex. ${ }^{18,19}$ Progestin-only ECPs have now largely replaced the older combined ECPs, because they are more effective, safe and causing fewer side effects.

Female health workers (nurses and midwives) are the integral part of our health care system. They are both service providers and health educators to the community. Large numbers of fertile age women are in contact with female health workers and they act as a reliable source of information. They have influence on women's contraceptive behaviour. Female health workers knowledge, attitudes and practices towards ECPs use can either promote or impede women's use of ECP. There was a paucity of such studies in our country. Therefore we plan to explore female health workers knowledge, attitude and practice towards ECP. We have carried out this study on female health workers (nurses and midwives) of a tertiary care hospital located in Karachi.

\section{METHODS}

A cross sectional survey was conducted at Kharadar General Hospital (KGH), Karachi. Data was collected from April to June 2016 after taking approval from ethics review committee (ERC) of KGH. Kharadar general hospital is a 250 bedded teaching hospital. It provides both teaching (School of nursing/ Post graduate trainee doctors) and health care (out patient department and in patient) services. It has sufficient female health care workers including consultants, doctors, nurses, midwives, technicians and students. Among them we have invited nurses $(\mathrm{N}=108)$ and midwives $(\mathrm{N}=94)$ to participate in this study (total=202). They were briefed about the objectives of the study. Total One hundred eighty seven (95 nurses and 92 midwives) participants who were consented to participate voluntarily in the study were included in the study.

Beside limited demographic variables (age, marital status, religion, education and clinical experience) a 26-item questionnaire was constructed to assess knowledge, attitudes and practice of study participants regarding ECP, based on literature review of similar studies conducted in other countries. ${ }^{1,20,21}$ Few questions were rephrased as per format of the questionnaire. Knowledge of ECP was assessed through twelve questions with yes, no and don't know options, Initial two questions (Ever heard about ECP and source of information) were not given score. Knowledge questions covers awareness, indication, time limit, side effects, effectiveness, safety, and mechanism of action of ECPs. Each correct answer was given one point and no point was given for wrong answers or for don't know options. Total knowledge scores (0-10, cut off score 5) were summed and then divided into two categories as adequate ( $>5$ score) or inadequate $(\leq 5$ score) knowledge of ECP.

Female health workers attitude was measured through eight items, rated on a four-point Likert scale as strongly disagree $(S D)=1$, disagree $(D)=2$, agree $(A)=3$, and strongly agree $(\mathrm{SA})=4$ except two negative items (religion is a major hurdle for ECP use and ECP might affect future pregnancy) were scored reversely $(\mathrm{SD}=4$, $\mathrm{D}=3, \mathrm{~A}=2$ and $\mathrm{SA}=1$ ). Likert scale reflected common deterrents to ECP use. The minimum score set for each respondent at 8 and maximum score at 32 with cut off score of 20 . Higher score $(>20)$ was indicative of positive attitude where as low score $(\leq 20)$ was indicative of a negative attitude. Participants practice required to state their prior experience regarding ECPs. It consists of another six statements with yes and no options.

Confidentiality of the participants was ensured through proper coding and masking the identification. Data entry and analysis was carried out by using Statistical Package for Social Science (SPSS) version 16.0. For categorical variables, frequencies and percentages were determined. Mean and standard deviation was computed for continuous variables like age. Categorical variables were compared by using Pearson chi-square test. A p value of $<0.05$ was considered statistically significant. 


\section{RESULTS}

Total 187 female health workers were participated in the study with participation rate of $92.6 \%$ (187/202). Among participants nurses were $95(50.8 \%)$ and midwives were $92(49.2 \%)$. Mean age of study participants was $23.5 \pm 7.31$ years. Majority participants $163(87.2 \%)$ were Muslim followed by Christian $22(11.8 \%)$ and Hindu 2 (1.1\%). Only $23(12.3 \%)$ participants were married. The average clinical experience of study participants was $2.7 \pm 5.40$ years. Among nurses it was $2.3 \pm 5.32$ years and in midwives $3.2 \pm 5.48$ years respectively (Table 1 ).

Table 1: Demographic characteristics of study participants $(\mathrm{N}=187)$.

\begin{tabular}{|c|c|c|c|}
\hline Characteristic & $\begin{array}{l}\text { Nurses } \\
(\mathrm{N}=95) \\
\mathbf{N}(\%)\end{array}$ & $\begin{array}{l}\text { Midwives } \\
(\mathbf{N}=92) \\
\mathbf{N}(\%)\end{array}$ & $\begin{array}{l}\text { Total } \\
(\mathrm{N}=187) \\
\mathbf{N}(\%)\end{array}$ \\
\hline $\begin{array}{l}\text { Mean age } \\
(\mathrm{SD})^{*}\end{array}$ & $22.97 \pm 7.60$ & $24.14 \pm 6.97$ & $23.5 \pm 7.31$ \\
\hline Minimum & 16 & 18 & 16 \\
\hline Maximum & 60 & 60 & 60 \\
\hline \multicolumn{4}{|l|}{ Marital Status } \\
\hline Single & $85(89.5)$ & $79(85.9)$ & $164(87.7)$ \\
\hline Married & $10(10.5)$ & $13(14.1)$ & $23(12.3)$ \\
\hline \multicolumn{4}{|l|}{ Religion } \\
\hline Islam & $79(83.2)$ & $84(91.3)$ & $163(87.2)$ \\
\hline Christian & $15(15.8)$ & $7(7.6)$ & $22(11.8)$ \\
\hline Hindu & $1(1.1)$ & $1(1.1)$ & $2(1.1)$ \\
\hline \multicolumn{4}{|l|}{ Education } \\
\hline $\begin{array}{l}\text { Matric/ } \\
\text { O level }\end{array}$ & 55 (57.9) & $56(60.9)$ & $111(59.4)$ \\
\hline $\begin{array}{l}\text { Intermediate/ } \\
\text { A level }\end{array}$ & $34(35.8)$ & 33 (35.9) & $67(35.8)$ \\
\hline Graduate & $6(6.3)$ & $3(3.3)$ & $9(4.8)$ \\
\hline $\begin{array}{l}\text { Working } \\
\text { experience } \\
\text { (Mean years) }\end{array}$ & $2.34 \pm 5.32$ & $3.15 \pm 5.48$ & $2.7 \pm 5.40$ \\
\hline Minimum & 0 & 0 & 0 \\
\hline Maximum & 40 & 39 & 40 \\
\hline
\end{tabular}

\section{Knowledge of ECPs}

Majority $123(65.8 \%)$ participants have already heard about ECPs and their main source of information was health personnel (doctors/ nurses/ pharmacist) 98 $(79.7 \%)$. Whereas $64(34.2 \%)$ did not hear about ECPs (Table 2). Only one-third $63(33.7 \%)$ of the study participants were aware that ECP is used after unprotected sex and nearly same proportion of participants $60(32.1 \%)$ were knew the correct time limit of 72 to 120 hours for administration of ECP after unprotected sex. Only 41 (21.9\%) study participants were aware that levonorgestrel is used as an ECP. Majority participants $131(70.0 \%)$ did not know the main side effect of ECP. More than half 108 (57.8\%) of participants were confused about the ECP mode of action as an abortifacient. Half of participants $95(50.8 \%)$ did not know the fact that ECP cannot protect against HIV/AIDS or other sexually Transmitted Diseases (STDs) (Table 2). Mean knowledge score of the study participants was $3.7 \pm 2.18$ (1-10, cut off score 5) (Table 4). Overall only $36(19.3 \%)$ participants had adequate (score >50\%) knowledge about ECP. Knowledge difference between nurses $17(17.9 \%)$ and midwives $19(20.7 \%)$ were not found significant $(\mathrm{r}=0.633, \quad \mathrm{P}>0.05)$. Participants characteristics associated with adequate knowledge were marital status $(\mathrm{r}=0.01, \mathrm{p}<0.05)$ and work experience $\geq 3$ years $(r=0.002, p<0.05)($ Table 5$)$.

\section{Attitudes towards ECPs}

The mean attitude score of the study participants was $20.9 \pm 2.68$ (8-32, cutoff score 20). More than half 103 $(54.9 \%)$ of the study participants had positive (score $>50 \%$ ) attitudes towards ECPs (Table 4). Proportion wise midwives $55(59.8 \%)$ have more positive attitude than nurses $48(50.5 \%)$ but statistically not significant $(\mathrm{r}=0.203, \mathrm{P}>0.05)$ (Table 5). Majority participants 118 $(63.1 \%)$ either agreed or strongly agreed that they would use ECPs for unwanted pregnancy (Table 3). Similarly majority participants $117(62 \%)$ participants thought that use of ECPs was safe. Significant number of participants $113(60.5 \%)$ were either disagreed or strongly disagreed to support the availability of ECPs without doctor's prescription. Two-third of participants 119 (63.6\%) also support ECP advertisement through electronic media (Table 3). Participant's characteristics were correlated with positive attitude but there was no significant association found (Table 5).

\section{Practice towards ECPs}

Regarding practice, 79 (42.5\%) of the participants, were supposed ECP as a routine contraceptive method and believed increased dose of routine contraceptive pills would work as ECP. Majority participants 113 (60.4\%) were not considered IUCD as effective EC method for preventing pregnancy within 120 hours of unprotected sex. Only $55(29.4 \%)$ participants were advised ECP to their clients with an average of one client in a month.

\section{DISCUSSION}

Emergency Contraceptive Pills (ECPs) are the only form of hormonal contraceptives that provide women a last chance to prevent pregnancy after unprotected sex. ${ }^{3,22}$ In Pakistan currently women have an average of 3.8 children. $^{23}$ Total fertility rate (TFR) was gradually decreasing over the last four decade from 6 to 4 children but reduction in fertility rate has not been accompanied by a concomitant reduction in unwanted pregnancies. 
Table 2: Female health worker's knowledge about emergency contraceptive pills $(\mathrm{N}=187)$.

\begin{tabular}{|c|c|c|c|c|}
\hline S. No & Items (Correct) & $\begin{array}{l}\text { Nurses }(\mathbf{N}=95) \\
\mathbf{N}(\%)\end{array}$ & $\begin{array}{l}\text { Midwives ( } \mathbf{N}=\mathbf{9 2}) \\
\mathbf{N}(\%)\end{array}$ & $\begin{array}{l}\text { Total }(\mathrm{N}=187) \\
\mathbf{N}(\%)\end{array}$ \\
\hline 1. & \multicolumn{4}{|l|}{ Have you ever heard about ECP*? } \\
\hline & Yes & $63(66.3)$ & $60(65.2)$ & $123(65.8)$ \\
\hline & No & $27(28.4)$ & $21(22.8)$ & $48(25.7)$ \\
\hline & Don't Know & $5(5.3)$ & $11(12.0)$ & $16(8.6)$ \\
\hline 2. & \multicolumn{4}{|l|}{ How did you know about ECP? } \\
\hline & Media/Internet & $4(4.2)$ & $8(8.7)$ & $12(6.4)$ \\
\hline & Health Personnel (doctors/ nurses/ pharmacist) & $53(55.8)$ & $45(48.9)$ & $98(52.4)$ \\
\hline & Fiends/ Family Members & $3(3.2)$ & $7(7.6)$ & $10(5.4)$ \\
\hline & Visit to FP Centre & $3(3.1)$ & $0(0.0)$ & $3(1.6)$ \\
\hline & Not Responded & $32(33.7)$ & $32(34.8)$ & $64(34.2)$ \\
\hline 3. & \multicolumn{4}{|l|}{ Is Levonorgestrel an ECP? (Yes) } \\
\hline & Yes & $23(24.2)$ & $18(19.6)$ & $41(21.9)$ \\
\hline & No & $13(13.7)$ & $20(21.7)$ & $33(17.7)$ \\
\hline & Don't Know & $59(62.1)$ & $54(58.7)$ & $113(60.4)$ \\
\hline 4. & \multicolumn{4}{|c|}{ After unprotected Sex do women use ECP to avoid pregnancy? (Yes) } \\
\hline & Yes & $27(28.4)$ & $36(39.1)$ & $63(33.7)$ \\
\hline & No & $40(42.1)$ & $37(40.2)$ & $77(41.2)$ \\
\hline & Don't Know & $28(29.5)$ & $19(20.7)$ & $47(25.1)$ \\
\hline 5. & \multicolumn{4}{|c|}{ Can Women use ECP within 72-120 hours after unprotected sex? (Yes) } \\
\hline & 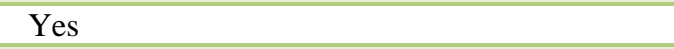 & $22(23.2)$ & $38(41.3)$ & $60(32.1)$ \\
\hline & No & $27(28.4)$ & $29(31.5)$ & $56(29.9)$ \\
\hline & Don't Know & $46(48.4)$ & $25(27.2)$ & $71(38.0)$ \\
\hline 6. & \multicolumn{4}{|c|}{ Is Menstrual irregularity the main side effect of ECP? (No) } \\
\hline & Yes & $58(61.1)$ & 43(46.7) & $101(54.0)$ \\
\hline & No & $18(18.9)$ & $38(41.3)$ & $56(30.0)$ \\
\hline & Don't Know & $19(20.0)$ & $11(12.0)$ & $30(16.0)$ \\
\hline 7. & \multicolumn{4}{|c|}{ Can a women use ECP every time when she has sex? (No) } \\
\hline & Yes & $32(33.7)$ & $33(35.9)$ & $65(34.8)$ \\
\hline & No & $42(44.2)$ & $40(43.5)$ & $82(43.8)$ \\
\hline & Don't Know & $21(22.1)$ & $19(20.7)$ & $40(21.4)$ \\
\hline 8. & \multicolumn{4}{|c|}{ Does ECP protect against HIV ${ }^{\dagger \dagger} / A I D S$ or other sexually transmitted disease? (No) } \\
\hline & Yes & $34(35.8)$ & 22(23.9) & $56(29.9)$ \\
\hline & No & $41(43.2)$ & $51(55.4)$ & $92(49.2)$ \\
\hline & Don’t Know & $20(21.1)$ & $19(20.7)$ & $39(20.9)$ \\
\hline 9. & \multicolumn{4}{|l|}{ Does pregnancy test require before taking ECP? (No) } \\
\hline & Yes & $42(44.2)$ & $40(43.5)$ & $82(43.9)$ \\
\hline & No & $32(33.7)$ & $36(39.1)$ & $68(36.4)$ \\
\hline & Don’t Know & $21(22.1)$ & $16(17.4)$ & $37(19.8)$ \\
\hline 10 & \multicolumn{4}{|c|}{ After taking ECP, do women expect next period within next month? (Yes) } \\
\hline & Yes & $42(44.2)$ & $47(51.1)$ & $89(47.6)$ \\
\hline & No & $28(29.5)$ & $23(25.0)$ & $51(27.3)$ \\
\hline & Don't Know & $25(26.3)$ & $22(23.9)$ & $47(25.1)$ \\
\hline 11. & \multicolumn{4}{|l|}{ Does ECP act as an abortifacient? (No) } \\
\hline & Yes & $21(22.1)$ & $22(23.9)$ & $43(23.0)$ \\
\hline & No & $38(40.0)$ & $41(44.6)$ & $79(42.2)$ \\
\hline & Don’t Know & $36(37.9)$ & $29(31.5)$ & $65(34.8)$ \\
\hline 12. & \multicolumn{4}{|l|}{ Does an IUCD ${ }^{* *}$ consider as an $\mathrm{EC}^{\dagger}$ method? (Yes) } \\
\hline & Yes & $38(40.0)$ & $32(34.8)$ & $70(37.4)$ \\
\hline & No & $38(40.0)$ & $43(46.7)$ & $81(43.3)$ \\
\hline & Don't Know & $19(20.0)$ & $17(18.5)$ & $36(19.3)$ \\
\hline $\begin{array}{l}{ }^{*} \mathrm{ECP}= \\
{ }_{* *} \mathrm{IUCI} \\
\dagger \mathrm{EC}= \\
{ }^{\dagger \dagger} \mathrm{HIV}= \\
{ }^{\mathrm{I}} \mathrm{AIDS}\end{array}$ & $\begin{array}{l}\text { mergency Contraceptive Pills } \\
\text { = Intrauterine contraceptive device } \\
\text { mergency Contraception } \\
\text { Human Immuno-deficiency virus } \\
\text { Acquired immune-deficiency disease syndrome }\end{array}$ & & & \\
\hline
\end{tabular}


Table 3: Female health worker's attitude towards emergency contraceptive pills $(\mathrm{N}=187)$.

\begin{tabular}{|c|c|c|c|c|}
\hline S No & Items & $\begin{array}{l}\text { Nurses }(\mathbf{N}=\mathbf{9 5}) \\
\mathbf{N}(\%)\end{array}$ & $\begin{array}{l}\text { Midwives ( } \mathbf{N}=\mathbf{9 2} \text { ) } \\
\mathbf{N}(\%)\end{array}$ & $\begin{array}{l}\text { Total }(\mathbf{N}=187) \\
\text { N }(\%)\end{array}$ \\
\hline \multirow[t]{5}{*}{1} & \multicolumn{4}{|c|}{ I Would prefer to use ECP for unwanted pregnancy } \\
\hline & Strongly disagree & $11(11.6)$ & $4(4.3)$ & $15(8.0)$ \\
\hline & Disagree & 21(22.1) & $33(35.9)$ & $54(28.9)$ \\
\hline & Agree & $55(57.9)$ & $48(52.2)$ & $103(55.1)$ \\
\hline & Strongly Agree & $8(8.4)$ & $7(7.6)$ & $15(8.0)$ \\
\hline \multirow[t]{5}{*}{2} & \multicolumn{4}{|c|}{ I Would recommend ECPs to others friend and family members } \\
\hline & Strongly disagree & $7(7.4)$ & $4(4.3)$ & $11(5.9)$ \\
\hline & Disagree & $26(27.4)$ & $24(26.1)$ & $50(26.7)$ \\
\hline & Agree & $53(55.8)$ & $57(62.0)$ & $110(58.8)$ \\
\hline & Strongly Agree & $9(9.5)$ & $7(7.6)$ & $16(8.6)$ \\
\hline \multirow[t]{5}{*}{3} & \multicolumn{4}{|l|}{ I suppose the ECP is safer for use } \\
\hline & Strongly disagree & $6(6.3)$ & $6(6.5)$ & $12(6.4)$ \\
\hline & Disagree & $32(33.7)$ & $26(28.3)$ & $58(31.0)$ \\
\hline & Agree & $46(48.4)$ & $55(59.8)$ & $101(54.0)$ \\
\hline & Strongly Agree & 11(11.6) & $5(5.4)$ & $16(8.6)$ \\
\hline \multirow[t]{5}{*}{4} & \multicolumn{4}{|c|}{ ECPs should be available easily without doctor prescription } \\
\hline & Strongly disagree & $30(31.6)$ & $9(9.8)$ & $39(20.9)$ \\
\hline & Disagree & $35(36.8)$ & $39(42.4)$ & 74(39.6) \\
\hline & Agree & $24(25.3)$ & $37(40.2)$ & 61(32.6) \\
\hline & Strongly Agree & $6(6.3)$ & $7(7.6)$ & $13(7.0)$ \\
\hline \multirow[t]{5}{*}{5} & \multicolumn{4}{|c|}{ Women should have the right to decide individually about the use of ECP } \\
\hline & Strongly disagree & $12(12.6)$ & $6(6.5)$ & $18(9.6$ \\
\hline & Disagree & $26(27.4)$ & $31(33.7)$ & $57(30.5)$ \\
\hline & Agree & $41(43.2)$ & $42(45.7)$ & $83(44.4)$ \\
\hline & Strongly Agree & $16(16.8)$ & $13(14.1)$ & $29(15.5)$ \\
\hline \multirow[t]{5}{*}{6} & \multicolumn{4}{|l|}{ Religion is a major hurdle for ECP use } \\
\hline & Strongly disagree & $14(14.7)$ & $12(13.0)$ & $26(13.9)$ \\
\hline & Disagree & $41(43.2)$ & $32(34.8)$ & 73(39.0) \\
\hline & Agree & $37(38.9)$ & $43(46.7)$ & $80(42.8)$ \\
\hline & Strongly Agree & $3(3.2)$ & $5(5.4)$ & $8(4.3)$ \\
\hline \multirow[t]{5}{*}{7} & \multicolumn{4}{|c|}{ I Would favour advertisement of ECPs through electronic media } \\
\hline & Strongly disagree & $7(7.4)$ & $7(7.6)$ & $14(7.5)$ \\
\hline & Disagree & $26(27.4)$ & $28(30.4)$ & $54(28.9)$ \\
\hline & Agree & $52(54.7)$ & $46(50.0)$ & $98(52.4)$ \\
\hline & Strongly Agree & $10(10.5)$ & $11(12.0)$ & $21(11.2)$ \\
\hline 8 & \multicolumn{4}{|l|}{ ECPs would affect pregnancy in the future } \\
\hline & Strongly disagree & $16(16.8)$ & 13(14.1) & $29(15.5)$ \\
\hline & Disagree & $41(43.2)$ & $53(57.6)$ & $94(50.3)$ \\
\hline & Agree & $27(28.4)$ & $18(19.6)$ & $45(24.1)$ \\
\hline & Strongly Agree & 11(11.6) & $8(8.7)$ & 19(10.2) \\
\hline
\end{tabular}

Table 4: Knowledge and attitude score of female health workers $(\mathrm{N}=187)$.

\begin{tabular}{|lllc|}
\hline Female Health Workers Score & Nurses $(\mathbf{N}=95)$ & $\begin{array}{l}\text { Midwives }(\mathbf{N}=92) \\
\mathbf{N}(\%)\end{array}$ & $\begin{array}{l}\text { Total }(\mathbf{N}=187) \\
\mathbf{N}(\%)\end{array}$ \\
\hline Knowledge Score (out of 10) & & & \\
\hline Mean \pm SD & & $4.1 \pm 2.13$ & $3.7 \pm 2.18$ \\
\hline Score $>5$ & $3.4 \pm 2.18$ & $19(20.7)$ & $36(19.3)$ \\
\hline Attitude Score (Range 8-32, cutoff 20) & $17(17.9)$ & & \\
\hline Mean \pm SD & & $21.1 \pm 2.40$ & $20.9 \pm 2.68$ \\
\hline Score $>$ 20 & $20.7 \pm 2.92$ & $55(59.8)$ & $103(54.9)$ \\
\hline *SD $=$ Standard Deviation & $48(50.5)$ & & \\
\hline
\end{tabular}


Table 5: Comparison of participant's characteristics with knowledge and attitude ( $\mathrm{N}=187)$.

\begin{tabular}{|c|c|c|c|c|c|c|}
\hline \multirow[b]{2}{*}{ Variables } & \multicolumn{2}{|l|}{ Knowledge } & \multirow[b]{2}{*}{ P-Value } & \multicolumn{2}{|l|}{ Attitude } & \multirow[b]{2}{*}{ P-Value } \\
\hline & $\begin{array}{l}\text { Adequate } \\
\mathbf{N}(\%)\end{array}$ & $\begin{array}{l}\text { Inadequate } \\
\mathbf{N}(\%)\end{array}$ & & $\begin{array}{l}\text { Positive } \\
\text { N }(\%)\end{array}$ & $\begin{array}{l}\text { Negative } \\
\mathbf{N}(\%)\end{array}$ & \\
\hline \multicolumn{7}{|l|}{ Female Health workers } \\
\hline Nurses $(\mathrm{N}=95)$ & $17(17.9)$ & $78(82.1)$ & \multirow{2}{*}{$\begin{array}{l}r=0.633 \\
P>0.05\end{array}$} & $48(50.5)$ & $47(49.5)$ & \multirow{2}{*}{$\begin{array}{l}r=0.203, \\
P>0.05\end{array}$} \\
\hline Midwives (N=92) & 19(20.7) & 73(79.3) & & $55(59.8)$ & $37(40.2)$ & \\
\hline \multicolumn{7}{|l|}{ Marital Status } \\
\hline Single $(N=164)$ & $27(16.5)$ & $137(83.5)$ & \multirow{2}{*}{$\begin{array}{l}r=0.01, \\
P<0.05\end{array}$} & $91(55.5)$ & 73(44.5) & \multirow{2}{*}{$\begin{array}{l}r=0.765 \\
P>0.05\end{array}$} \\
\hline Married (N=23) & $9(39.1)$ & $14(60.9)$ & & $12(52.2)$ & $11(47.8)$ & \\
\hline \multicolumn{7}{|l|}{ Religion } \\
\hline $\operatorname{Muslim}(\mathrm{N}=163)$ & $33(20.2)$ & $130(79.8)$ & \multirow{2}{*}{$\mathrm{NA}^{*}$} & $94(57.7)$ & $69(42.3)$ & \multirow{2}{*}{$\begin{array}{l}r=0.064 \\
P>0.05\end{array}$} \\
\hline Non-muslim $(\mathrm{N}=24)$ & $5(20.8)$ & $19(79.2)$ & & $9(37.5)$ & $15(62.5$ & \\
\hline \multicolumn{7}{|l|}{ Education } \\
\hline Matric $(\mathrm{N}=111)$ & $17(15.3)$ & 94(84.7) & \multirow{2}{*}{$\begin{array}{l}r=0.09 \\
P>0.05\end{array}$} & $62(55.9)$ & $49(44.1)$ & \multirow{2}{*}{$\begin{array}{l}r=0.797 \\
P>0.05\end{array}$} \\
\hline Intermediate or higher $(\mathrm{N}=76)$ & 19(25.0) & $57(75.0)$ & & $41(53.9)$ & $35(46.1)$ & \\
\hline \multicolumn{7}{|l|}{ Work experience } \\
\hline$<3$ years $(\mathrm{N}=129)$ & $17(13.2)$ & $112(86.8)$ & \multirow{2}{*}{$\begin{array}{l}r=0.002 \\
P<0.05\end{array}$} & $73(56.6)$ & $56(43.4)$ & \multirow{2}{*}{$\begin{array}{l}r=0.536 \\
P>0.05\end{array}$} \\
\hline$\geq 3$ years $(\mathrm{N}=58)$ & $19(32.8)$ & $39(67.2)$ & & $30(51.7)$ & $28(48.3)$ & \\
\hline
\end{tabular}

Estimated 29 of every 1,000 women of reproductive age still seek to terminate their unwanted or mistimed pregnancies through unsafe means and expose to abortion related morbidity and mortality. ${ }^{24}$

General awareness of ECP among study female health workers was $65.8 \%$ and their main source of information $(52.4 \%)$ was health personnel including doctors, nurses, and pharmacist. Jackson R, et al also reported the same source of information on ECP through health care providers. ${ }^{25}$ Study reported other source of information of ECP such as friends and family members, similar source of information on ECP was reported by Kongnyuy, et al. ${ }^{1}$

The important finding of this study was identification of inadequate knowledge of majority participants (81\%) regarding ECP and significant participants (45\%) have negative attitudes towards ECP without significant difference between both groups (nurses and midwives) of female health workers. Previous study conducted in Pakistan among lady health supervisors, who provides family planning services at the doorsteps of people, also reported similar finding of insufficient knowledge and strong negative attitudes towards ECP use. ${ }^{26}$ Similar results were reported in other developing countries including India, Nepal, and south Africa. ${ }^{27-29}$ In contrast to our finding high level of knowledge was reported among nursing student by a Brazilian study (86-96\%) and in Iranian study $(93.5 \%) .{ }^{21,30}$ Most likely reason for this low knowledge among female health workers was that in nursing and midwifery curriculum, methods of contraception have been teaching superficially but neither providing formal training nor focusing the methods of emergency contraception. Mostly female health workers learn about ECP during their clinical experience in the hospital particularly while working in reproductive health unit. Study also reported that participant's clinical experience was significantly associated with adequate knowledge.

In general, the rates of emergency contraception use were lowest in Asia and Africa $(<3 \%)$ even low in the developed countries, where the ECP is available to most women of childbearing age without prescription, such as in the United States $(11 \%)$ and France $(17 \%)$, respectively. ${ }^{31,32}$ In Pakistan the proportion of married women of reproductive age (15-49 years) who had heard of ECP was $17.7 \%$ and ever used of ECP was only $0.9 \% .^{33}$ This very low rate of ECP use in Pakistan reflect the lack of capacity of health workers (health system) regarding ECP knowledge and counselling skills to the women. Low ECP knowledge and negative attitude of female health workers would create negative impact on preventing unwanted pregnancies through emergency contraceptives methods.

Study also reported that two-third $(68 \%)$ of participants did not know the use of ECP correct time limit of 72 to 120 hours after unprotected sex during which potential contraception could be used effectively. Previous studies have also confirmed that ECP is effective upto120 hours after unprotected sex. ${ }^{34}$ Previous studies also reported the failure rate of ECP at 72 hours is approximately $4 \%$ which increases up to $10-50 \%$ at five days. ${ }^{35,36}$ More than one-third $(34.8 \%)$ participants thought that ECP can be used on regular basis whenever have a sex. Though ECP is intended for occasional or emergency use only and not as a regular contraception. ${ }^{37}$ The currently 
available evidence indicates that ECP is safe and effective even when used several times. ${ }^{38,39}$ Study reported that more than half $(57.8 \%)$ of participants did not know that emergency contraceptive pills do not cause abortion. As the misconception of ECP as abortifacient, the female health workers are reluctant to use or prescribe ECPs. Therefore only $29 \%$ participants ever advised ECPs to their clients.

Study sample was limited because study was based on female health workers (nurses and midwives) of a single hospital. Therefore study results were not extrapolated on general population. Female doctors and male health workers of the study hospital were not included in the survey due to small in numbers. Majority $(90 \%)$ participants were unmarried therefore; we were not asked the leading question from the participants that have you ever used ECP.

\section{CONCLUSION}

In conclusion, the study identified the gap in Knowledge about ECP and significant negative attitude towards ECP among the female health workers. There is a need to improve female health workers knowledge about ECP and change their attitudes through providing formal training and exposure on emergency contraception practices. Also remove their misconception about the use and safety of ECP which can contribute to prevent unwanted pregnancies and abortion.

\section{ACKNOWLEDGEMENT}

We were grateful to the administration of Kharader General Hospital for their continuous support. We would like to thank the school of nursing and nursing staff matron for providing study participants. We were also thankful to all female health workers of Kharader General Hospital for being participated in the study voluntarily.

Funding: Financial support for the study was provided by the administrative management of Kharader General Hospital, Karachi

Conflict of interest: None declared

Ethical approval: The study was approved by the Institutional Ethics Committee

\section{REFERENCES}

1. Kongnyuy EJ, Ngassa P, Fomulu N, Wiysonge CS, Kouam L, Doh AS. A survey of knowledge, attitudes and practice of emergency contraception among university students in Cameroon. BMC Emergency Medicine. 2007;7:1-7.

2. World Health Organization: A Tabulation of Available Data on the Frequency and Mortality of Unsafe abortion.2nd edition. WHO Division of Family Health, Maternal Health and Safe Motherhood Programme, Geneva; 1994.
3. Grimes DA, Benson J, Singh S, Romero M, Ganatra B, Okonofua FE, et al. Unsafe abortion: the preventable pandemic. Lancet. 2006;368:1908-19.

4. World Health Organization: Complications of abortion: Technical and managerial guidelines for prevention and treatment Guidelines. Geneva; 1995.

5. Henshaw SK, Morrow E: Induced Abortion: A World Review. The Alan Guttmacher Institute Review;1990.

6. Popov AA. Family planning and induced abortion in the USSR: Basic health and demographic characteristics. Studies Fam Plan. 1991;22:368-77.

7. Sathar Z, Singh S, Fikree FF. Estimating the Incidence of Abortion in Pakistan. Stud Fam Plann. 2007;38(1):11-22.

8. Duncan G, Harper C, Ashwell E, Mant D, Buchan H, Jones L. Termination of pregnancy: lessons for prevention. Br J Fam Plann. 1990;15:112-7.

9. International Consortium for Emergency Contraception: Emergency Contraceptive Pills. Washington, DC $2^{\text {nd }}$ edition; 2004.

10. Marions L, Hultenby K, Lindell I, Sun X, Ståbi B, Danielsson GK. Emergency contraception with mifepristone and levonorgestrel: mechanism of action. Obstet Gynecol. 2002;100:65-71.

11. Marions L, Cekan SZ, Bygdeman M, GemzellDanielsson K. Effect of emergency contraception with levonorgestrel or mifepristone on ovarian function. Contraception. 2004;69:373-7.

12. Okewole IA, Arowojolu AO, Odusoga OL, Oloyede $\mathrm{OA}$, Adeleye OA, Salu J, et al. Effect of single administration of levonorgestrel on the menstrual cycle. Contraception. 2007;75:372-7.

13. Croxatto HB, Devoto L, Durand M, Ezcurra E, Larrea F, Nagle C, et al. Mechanism of action of hormonal preparations used for emergency contraception: a review of the literature. Contraception. 2001;63:111-21.

14. Croxatto HB, Ortiz ME, Müller AL. Mechanisms of action of emergency contraception. Steroids. 2003;68:1095-8.

15. David G, Weismiller A. Emergency Contraception. Am Fam Physician. 2004;70:707-14.

16. Yuzpe AA, Lancee WJ. Ethinylestradiol and dlnorgestrel as a postcoital contraceptive. Fertility and Sterility. 1977;28(9):932.

17. La Valleur J. Emergency contraception. Obstet Gynecol Clin North Am. 2000;27:817-39.

18. von Hertzen H, Piaggio G, Ding J, Chen J, Song S, Bártfai $\mathrm{G}$, et al. Low dose mifepristone and two regimens of levonorgestrel for emergency contraception: a WHO multicentre randomised trial. Lancet. 2002;360:1803-10.

19. Arowojolu AO, Okewole IA, Adekunle AO. Comparative evaluation of the effectiveness and safety of two regimens of levonorgestrel for emergency contraception in Nigerians. Contraception. 2002;66:269-73.

20. Shelat PR, Hihoriya NH, Kumbar S. Knowledge and attitude towards the use of emergency contraceptive 
pills among college students. Int J Basic Clin Pharmacol. 2012 Oct;1(2):77-84.

21. Veloso DL, Peres VC, Lopes JS, Salge AK, Guimarães JV. Emergency contraception: knowledge and attitudes of nursing students. Rev Gaúcha Enferm. 2014 Jun;35(2):33-9.

22. Trussell J, Raymond E G. Emergency Contraception: A Last Chance to Prevent Unintended Pregnancy; 2009. Available at http://ec.princeton.edu/questions/ec-review.pdf. Accessed 19 June 2016.

23. Pakistan demographic and health survey; 2012-2013. National Institute of Population Studies Islamabad, Pakistan. Available at http://www.nips.org.pk. Accessed 19 June 2016.

24. Unwanted pregnancy and post abortion complications in Pakistan: findings from a national study. October 2004, pop council. (Online) Cited 2008 April. Available at http://www.popcouncil.org/projects/RH_PakResearc hUnwantedPreg.html. Accessed June 192016.

25. Jackson R, Schwarz EB, Freedman L, Darney P. Knowledge and willingness to use emergency contraception among low-income post-partum women. Contraception. 2000;61:351-7.

26. Mir AS, Malik R. Emergency contraceptive pills: Exploring the knowledge and attitudes of community health workers in a developing Muslim country. N Am J Med Sci. 2010 Aug;2(8):359-64.

27. Nivedita K, Shanthini FN. Knowledge, Attitude and Practice of Emergency Contraception on Nursing Personnel. Journal of Clinical and Diagnostic Research. 2014 Sep;8(9):20-22.

28. Thapa B. Knowledge and attitude regarding EC among nursing personnel. JCMC. 2013;3(3):46-50.

29. Smit J, McFadyen L, Beksinska M, de Pinho H, Morroni C, Mqhayi M. Emergency contraception in South Africa: knowledge, attitudes, and use among public sector primary healthcare clients. Contraception. 2001;64:333-7.

30. Sharjabad FN, Hajivandi A, Rayani M. Knowledge, Attitude, and Practice about Emergency
Contraception among Health Staff in Bushehr State, South of Iran. Global Journal of Health Science. 2014;6(1):52-60.

31. Daniels K, Jones J, Abma J. Use of emergency contraception among women aged 15-44: United States, 2006-2010, NCHS Data Brief, 2013:112.

32. Moreau C, Bajos N, Trussell J. The impact of pharmacy ac-cess to emergency contraceptive pills in France, Contraception. 2006;73(6):602-08.

33. Palermo T, Bleck J, Westley E. Knowledge and Use of Emergency Contraception: A Multicountry Analysis. Int Perspect Sex Reprod Health. 2014 Jun;40(2):79-86.

34. Schwarz EB, Gerbert B, Gonzales R. Need for emergency contraception in urgent care settings. Contraception. 2007;75:285-88.

35. Piaggio G, von Hertzen H, Grimes DA, VanLook PF. Timing of emergency contraception with levonorgestrel or the Yuzpe regimen. Task force on post-ovulatory methods of fertility regulation. Lancet. 1999;353:721.

36. Trussell J, Rodríguez G, Ellertson C. Updated estimates of the effectiveness of the Yuzpe regimen of Emergency contraception. Contraception. 1999;59:147-51.

37. Van Look PFA, von Hertzen H. Emergency contraception. British Medical Bulletin. 1993;49:158-70.

38. Shelton, JD. Repeat emergency contraception: facing our fears. Contraception. 2002;66(1):15.

39. Abuabara K, Becker D, Ellertson C, Blanchard K, Schiavon R, Garcia SG. As often as needed: appropriate use of emergency contraceptive pills. Contraception. 2004;69(4):339-42.

Cite this article as: Ahmed M, Naz F, Iqbal K, Memon A. Knowledge, attitude and practice regarding emergency contraceptive pills among female health workers of a tertiary care hospital in Karachi. Int J Reprod Contracept Obstet Gynecol 2016;5:4248-55. 